\title{
First measurement of the forward-backward asymmetry in bottom-quark pair production at high mass
}

T. Aaltonen, ${ }^{21}$ S. Amerio, ${ }^{39 a, 39 b}$ D. Amidei, ${ }^{31}$ A. Anastassov,${ }^{15, w}$ A. Annovi, ${ }^{17}$ J. Antos,${ }^{12}$ G. Apollinari, ${ }^{15}$ J. A. Appel, ${ }^{15}$ T. Arisawa, ${ }^{52}$ A. Artikov, ${ }^{13}$ J. Asaadi, ${ }^{47}$ W. Ashmanskas, ${ }^{15}$ B. Auerbach, ${ }^{2}$ A. Aurisano, ${ }^{47}$ F. Azfar, ${ }^{38}$ W. Badgett,,${ }^{15}$ T. Bae, ${ }^{25}$ A. Barbaro-Galtieri, ${ }^{26}$ V. E. Barnes, ${ }^{43}$ B. A. Barnett ${ }^{23}$ P. Barria,${ }^{41 a, 41 \mathrm{c}}$ P. Bartos ${ }^{12}$ M. Bauce,${ }^{39 a, 39 b}$ F. Bedeschi, ${ }^{41 a}$ S. Behari, ${ }^{15}$ G. Bellettini, ${ }^{4 \mathrm{a}, 41 \mathrm{~b}}$ J. Bellinger, ${ }^{54}$ D. Benjamin, ${ }^{14}$ A. Beretvas, ${ }^{15}$ A. Bhatti, ${ }^{45}$ K. R. Bland, ${ }^{5}$ B. Blumenfeld ${ }^{23}$ A. Bocci, ${ }^{14}$

A. Bodek ${ }^{44}$ D. Bortoletto, ${ }^{43}$ J. Boudreau, ${ }^{42}$ A. Boveia, ${ }^{11}$ L. Brigliadori, ${ }^{6,}, 6 \mathrm{~b}$ C. Bromberg, ${ }^{32}$ E. Brucken ${ }^{21}$ J. Budagov, ${ }^{13}$ H. S. Budd, ${ }^{44}$ K. Burkett, ${ }^{15}$ G. Busetto, ${ }^{39 a, 39 b}$ P. Bussey, ${ }^{19}$ P. Butti, ${ }^{41 a, 41 b}$ A. Buzatu, ${ }^{19}$ A. Calamba, ${ }^{10}$ S. Camarda, M. Campanelli, ${ }^{28}$ F. Canelli, ${ }^{11, e e}$ B. Carls ${ }^{22}$ D. Carlsmith, ${ }^{54}$ R. Carosi, ${ }^{41 a}$ S. Carrillo, ${ }^{16,1}$ B. Casal,,${ }^{9, j}$ M. Casarsa, ${ }^{48 a}$ A. Castro, ${ }^{6 a, 6 b}$ P. Catastini, ${ }^{20}$ D. Cauz ${ }^{48 a, 48 b, 48 c}$ V. Cavaliere, ${ }^{22}$ A. Cerri, ${ }^{26, e}$ L. Cerrito, ${ }^{28, r}$ Y. C. Chen, ${ }^{1}$ M. Chertok, ${ }^{7}$ G. Chiarelli, ${ }^{41 a}$ G. Chlachidze, ${ }^{15}$ K. Cho, ${ }^{25}$ D. Chokheli, ${ }^{13}$ A. Clark, ${ }^{18}$ C. Clarke,${ }^{53}$ M. E. Convery, ${ }^{15}$ J. Conway, ${ }^{7}$ M. Corbo, ${ }^{15, z}$ M. Cordelli, ${ }^{17}$ C. A. Cox, ${ }^{7}$ D. J. Cox,${ }^{7}$ M. Cremonesi, ${ }^{4 a}$ D. Cruz, ${ }^{47}$ J. Cuevas, ${ }_{9, y}$ R. Culbertson, ${ }^{15}$ N. d'Ascenzo, ${ }^{15, v}$ M. Datta, ${ }^{15, h h}$ P. de Barbaro, ${ }^{44}$ L. Demortier, ${ }^{45}$ M. Deninno ${ }^{6 \mathrm{a}}$ M. D’Errico, ${ }^{39 \mathrm{a}, 39 \mathrm{~b}}$ F. Devoto, ${ }^{21}$ A. Di Canto, ${ }^{41 \mathrm{a}, 41 \mathrm{~b}}$ B. Di Ruzza ${ }_{15, p}^{1}$ J. R. Dittmann, ${ }^{5}$ S. Donati, ${ }^{41 a, 41 b}$ M. D'Onofrio, ${ }^{27}$ M. Dorigo, ${ }^{48 a}, 48 \mathrm{~d}$ A. Driutti, ${ }^{48 a, 48 b, 48 c}$ K. Ebina, ${ }^{52}$ R. Edgar,${ }^{31}$ A. Elagin, ${ }^{47}$ R. Erbacher ${ }^{7}$ S. Errede, ${ }^{22}$ B. Esham, ${ }^{22}$ S. Farrington, ${ }^{38}$ J. P. Fernández Ramos, ${ }^{29}$ R. Field, ${ }^{16}$ G. Flanagan,${ }^{15, t}$ R. Forrest, ${ }^{7}$ M. Franklin, ${ }^{20}$ J. C. Freeman,${ }^{15}$ H. Frisch, ${ }^{11}$ Y. Funakoshi, ${ }^{52}$ C. Galloni,${ }^{41 a, 41 b}$ A. F. Garfinkel, ${ }^{43}$ P. Garosi, ${ }^{41 a, 41 c}$ H. Gerberich ${ }^{22}$ E. Gerchtein, ${ }^{15}$ S. Giagu, ${ }^{46 a}$ V. Giakoumopoulou, ${ }^{3}$ K. Gibson, ${ }^{42}$ C. M. Ginsburg, ${ }^{15}$ N. Giokaris, ${ }^{3}$ P. Giromini, ${ }^{17}$ V. Glagolev, ${ }^{13}$ D. Glenzinski, ${ }^{15}$ M. Gold,${ }^{34}$ D. Goldin ${ }^{47}$ A. Golossanov, ${ }^{15}$ G. Gomez, ${ }^{9}$ G. Gomez-Ceballos, ${ }^{30}$ M. Goncharov ${ }^{30}$ O. González López, ${ }^{29}$ I. Gorelov, ${ }^{34}$ A. T. Goshaw,${ }^{14}$ K. Goulianos, ${ }^{45}$ E. Gramellini, ${ }^{6 a}$ C. Grosso-Pilcher, ${ }^{11}$ R. C. Group, ${ }^{51,15}$ J. Guimaraes da Costa, ${ }^{20}$ S. R. Hahn, ${ }^{15}$ J. Y. Han ${ }^{44}$ F. Happacher, ${ }^{17}$ K. Hara, ${ }^{49}$ M. Hare, ${ }^{50}$ R. F. Harr ${ }^{53}$ T. Harrington-Taber, ${ }^{15, m}$ K. Hatakeyama ${ }^{5}$ C. Hays, ${ }^{38}$ J. Heinrich, ${ }^{40}$ S. Henry, ${ }_{55}$ M. Herndon, ${ }^{54}$ A. Hocker, ${ }^{15}$ Z. Hong ${ }^{47}$ W. Hopkins, ${ }^{15, \mathrm{f}}$ S. Hou, ${ }_{15}$ R. E. Hughes, ${ }^{35}$ U. Husemann, ${ }^{55}$ M. Hussein, ${ }^{32, c c}$ J. Huston, ${ }^{32}$ G. Introzzi, ${ }^{41 a, 41 e, 41 \mathrm{f}}$ M. Iori, ${ }^{46 a, 46 b}$ A. Ivanov, ${ }^{7, o}$ E. James, ${ }^{15}$ D. Jang, ${ }^{10}$ B. Jayatilaka, ${ }^{15}$ E. J. Jeon, ${ }^{25}$ S. Jindariani, ${ }^{15}$ M. Jones, ${ }^{43}$ K. K. Joo, ${ }^{25}$ S. Y. Jun, ${ }^{10}$ T. R. Junk, ${ }^{15}$ M. Kambeitz, ${ }^{24}$ T. Kamon, ${ }^{25,47}$ P. E. Karchin, ${ }^{53}$ A. Kasmi, ${ }^{5}$ Y. Kato ${ }^{37, n}$ W. Ketchum, ${ }^{11, i i}$ J. Keung, ${ }^{40}$ B. Kilminster, ${ }^{15, e e}$ D. H. Kim, ${ }^{25}$ H. S. Kim, ${ }^{15, b b}$ J. E. Kim, ${ }^{25}$ M. J. Kim, ${ }^{17}$ S. H. Kim, ${ }^{49}$ S. B. Kim, ${ }^{25}$ Y. J. Kim, ${ }^{25}$ Y. K. Kim, ${ }^{11}$ N. Kimura, ${ }^{52}$ M. Kirby, ${ }^{15}$ K. Knoepfel, ${ }^{15}$ K. Kondo, ${ }^{52, *}$ D. J. Kong, ${ }^{25}$ J. Konigsberg, ${ }^{16}$ A. V. Kotwal, ${ }^{14}$ M. Kreps ${ }^{24}$ J. Kroll, ${ }^{40}$ M. Kruse, ${ }^{14}$ T. Kuhr, ${ }^{24}$ M. Kurata, ${ }^{49}$ A. T. Laasanen, ${ }^{43}$ S. Lammel, ${ }^{15}$ M. Lancaster, ${ }^{28}$ K. Lannon, ${ }^{35, x}$ G. Latino ${ }^{41 a, 41 c}$ H. S. Lee, ${ }^{25}$ J. S. Lee,${ }^{25}$ S. Leo, ${ }^{22}$ S. Leone,${ }^{41 a}$ J. D. Lewis, ${ }^{15}$ A. Limosani ${ }^{14, s}$ E. Lipeles, ${ }^{40}$ A. Lister, ${ }^{18, a}$ H. Liu,${ }^{51}$ Q. Liu ${ }^{43}$ T. Liu, ${ }^{15}$ S. Lockwitz, ${ }^{55}$ A. Loginov, ${ }^{55}$ D. Lucchesi ${ }^{39 a, 39 b}$ A. Lucà, ${ }^{17}$ J. Lueck ${ }^{24}$ P. Lujan, ${ }^{26}$ P. Lukens, ${ }^{15}$ G. Lungu, ${ }^{45}$ J. Lys, ${ }^{26}$ R. Lysak, ${ }^{12, d}$ R. Madrak,${ }^{15}$ P. Maestro, ${ }^{41 \mathrm{a}, 41 \mathrm{c}}$ S. Malik, ${ }^{45}$ G. Manca, ${ }^{27, \mathrm{~b}}$ A. Manousakis-Katsikakis, ${ }^{3}$ L. Marchese, ${ }^{6 a, j j}$ F. Margaroli, ${ }^{46 a}$ P. Marino, ${ }^{41 a, 41 \mathrm{~d}}$ K. Matera, ${ }^{22}$ M. E. Mattson, ${ }^{53}$ A. Mazzacane, ${ }^{15}$ P. Mazzanti, ${ }^{6 a}$ R. McNulty, ${ }^{27, i}$ A. Mehta, ${ }^{27}$ P. Mehtala, ${ }^{21}$ C. Mesropian, ${ }^{45}$ T. Miao, ${ }^{15}$ D. Mietlicki, ${ }^{31}$ A. Mitra, ${ }^{1}$ H. Miyake, ${ }^{49}$ S. Moed, ${ }^{15}$ N. Moggi, ${ }^{6 a}$ C. S. Moon, ${ }^{15, z}$ R. Moore, ${ }^{15, f f, g g}$ M. J. Morello, ${ }^{41 a, 41 d}$ A. Mukherjee, ${ }^{15}$ Th. Muller, ${ }^{24}$ P. Murat, ${ }^{15}$ M. Mussini, ${ }^{6 a, 6 b}$ J. Nachtman, ${ }^{15, m}$ Y. Nagai ${ }^{49}$ J. Naganoma ${ }^{52}$ I. Nakano, ${ }^{36}$ A. Napier, ${ }^{50}$ J. Nett, ${ }^{47}$ C. Neu, ${ }^{51}$ T. Nigmanov, ${ }^{42}$ L. Nodulman, ${ }^{2}$ S. Y. Noh,${ }^{25}$ O. Norniella, ${ }^{22}$ L. Oakes,${ }^{38}$ S. H. Oh, ${ }^{14}$ Y. D. Oh,${ }^{25}$ I. Oksuzian, ${ }^{51}$ T. Okusawa,${ }^{37}$ R. Orava, ${ }^{21}$ L. Ortolan, ${ }^{4}$ C. Pagliarone, ${ }^{48 a}$ E. Palencia,,${ }^{9}$ e P. Palni, ${ }^{34}$ V. Papadimitriou, ${ }^{15}{ }^{2}$ W. Parker, ${ }^{54}$ G. Pauletta, ${ }^{4 a, 48 b, 48 c}$ M. Paulini ${ }^{10}$ C. Paus, ${ }^{30}$ T. J. Phillips, ${ }^{14}$ G. Piacentino, ${ }^{15, q}$ E. Pianori, ${ }^{40}{ }^{13}$. Pilot, ${ }^{7}$ K. Pitts,${ }^{22}$ C. Plager, ${ }^{8}$ L. Pondrom,${ }^{54}$ S. Poprocki, ${ }^{15, \mathrm{f}}$ K. Potamianos, ${ }^{26}$ A. Pranko, ${ }^{26}$ F. Prokoshin, ${ }^{13, \text { aa }}$ F. Ptohos, ${ }^{17, \mathrm{~g}}$ G. Punzi, ${ }^{41 \mathrm{a}, 41 \mathrm{~b}}$ I. Redondo Fernández, ${ }^{29}$ P. Renton, ${ }^{38}$ M. Rescigno, ${ }^{46 a}$ F. Rimondi, ${ }^{6 a,}{ }^{*}$ L. Ristori, ${ }^{41,15}$ A. Robson, ${ }^{19}$ T. Rodriguez, ${ }^{40}$ S. Rolli, ${ }^{50, h}$ M. Ronzani, ${ }^{41 \mathrm{a}, 41 \mathrm{~b}}$ R. Roser, ${ }^{15}$ J. L. Rosner, ${ }^{11}$ F. Ruffini, ${ }^{41 \mathrm{a}, 41 \mathrm{c}}$ A. Ruiz, ${ }^{9}$ J. Russ, ${ }^{10}$ V. Rusu, ${ }^{15}$ W. K. Sakumoto, ${ }^{44}$ Y. Sakurai, ${ }^{52}$ L. Santi, ${ }^{48 a, 48 b, 48 c}$ K. Sato, ${ }^{49}$ V. Saveliev, ${ }^{15, v}$ A. Savoy-Navarro, ${ }^{15, z}$ P. Schlabach, ${ }^{15}$ E. E. Schmidt ${ }^{15}$ T. Schwarz, ${ }^{31}$

L. Scodellaro, ${ }^{9}$ F. Scuri, ${ }^{41 \mathrm{a}}$ S. Seidel, ${ }^{34}$ Y. Seiya, ${ }^{37}$ A. Semenov, ${ }^{13}$ F. Sforza, ${ }^{41 a, 41 \mathrm{~b}}$ S. Z. Shalhout, ${ }^{7}$ T. Shears, ${ }^{27}$ P. F. Shepard, ${ }^{42}$ M. Shimojima, ${ }^{49, \mathrm{u}}$ M. Shochet, ${ }^{11}$ I. Shreyber-Tecker ${ }^{33}$ A. Simonenko, ${ }^{13}$ K. Sliwa, ${ }^{50}$ J. R. Smith, ${ }^{7}$ F. D. Snider, ${ }^{15}$ H. Song ${ }^{42}$ V. Sorin, ${ }^{4}$ R. St. Denis, ${ }^{19, *}$ M. Stancari, ${ }^{15}$ D. Stentz, ${ }^{15, w}$ J. Strologas,${ }^{34}$ Y. Sudo,${ }^{49}$ A. Sukhanov, ${ }^{15}$ I. Suslov, ${ }^{13}$ K. Takemasa ${ }^{49}$ Y. Takeuchi, ${ }^{49}$ J. Tang, ${ }^{11}$ M. Tecchio ${ }^{31}$ P. K. Teng, ${ }^{1}$ J. Thom $,{ }_{15},{ }^{1}$ E. Thomson,${ }^{40}$ V. Thukral, ${ }^{47}$ D. Toback, ${ }^{47}$ S. Tokar, ${ }^{12}$ K. Tollefson, ${ }^{32}$ T. Tomura, ${ }^{49}$ D. Tonelli, ${ }^{15, \mathrm{e}} \mathrm{S}$. Torre, ${ }^{17} \mathrm{D}$. Torretta, ${ }^{15} \mathrm{P}$. Totaro, ${ }^{39 \mathrm{a}} \mathrm{M}$. Trovato, ${ }^{41 \mathrm{a}, 41 \mathrm{~d}} \mathrm{~F}$. Ukegawa ${ }^{49} \mathrm{~S}$. Uozumi ${ }^{25}$ F. Vázquez, ${ }^{16,1}$ G. Velev, ${ }^{15}$ C. Vellidis, ${ }_{1}^{15}$ C. Vernieri, ${ }^{41 a, 41 d}$ M. Vidal,${ }^{43}$ R. Vilar, ${ }^{9}$ J. Vizán, ${ }^{9, d d}$ M. Vogel, ${ }^{34}$ G. Volpi, ${ }^{17}$ P. Wagner, ${ }^{40}$ R. Wallny, ${ }^{15, j}$ S. M. Wang, ${ }^{1}$ D. Waters ${ }^{28}$ W. C. Wester III ${ }^{15}$ D. Whiteson, ${ }^{40, c}$ A. B. Wicklund, ${ }^{2}$ S. Wilbur, ${ }^{7}$ H. H. Williams, ${ }^{40}$ J. S. Wilson, ${ }^{31}$ P. Wilson, ${ }^{15}$ B. L. Winer, ${ }^{35}$ P. Wittich, ${ }^{15,}{ }^{2}$ S. Wolbers, ${ }^{15} \mathrm{H}$. Wolfe, ${ }^{35}$ T. Wright,${ }^{31} \mathrm{X}$. Wu, ${ }^{18}$ Z. Wu, ${ }^{5}$ K. Yamamoto, ${ }^{37}$ D. Yamato, ${ }^{37}$ T. Yang, ${ }^{15}$ U. K. Yang, ${ }^{25}$ Y. C. Yang, ${ }^{25}$ W.-M. Yao, ${ }^{26}$ G. P. Yeh, ${ }^{15}$ K. Yi, ${ }^{15, m}$ J. Yoh, ${ }^{15}$ K. Yorita, ${ }^{52}$ T. Yoshida, ${ }^{37, k}$ G. B. Yu, ${ }^{14}$ I. Yu, ${ }^{25}$ A. M. Zanetti, ${ }^{4 a}$ Y. Zeng, ${ }^{14}$ C. Zhou, ${ }^{14}$ and S. Zucchelli ${ }^{6 a, 6 b}$

(CDF Collaboration)

${ }^{1}$ Institute of Physics, Academia Sinica, Taipei, Taiwan 11529, Republic of China ${ }^{2}$ Argonne National Laboratory, Argonne, Illinois 60439, USA 
${ }^{3}$ University of Athens, 15771 Athens, Greece

${ }^{4}$ Institut de Fisica d'Altes Energies, ICREA, Universitat Autonoma de Barcelona, E-08193 Bellaterra (Barcelona), Spain

${ }^{5}$ Baylor University, Waco, Texas 76798, USA

${ }^{6 a}$ Istituto Nazionale di Fisica Nucleare Bologna, I-40126 Bologna, Italy

${ }^{6 \mathrm{~b}}$ University of Bologna, I-40127 Bologna, Italy

${ }^{7}$ University of California, Davis, Davis, California 95616, USA

${ }^{8}$ University of California, Los Angeles, Los Angeles, California 90024, USA

${ }^{9}$ Instituto de Fisica de Cantabria, CSIC-University of Cantabria, 39005 Santander, Spain

${ }^{10}$ Carnegie Mellon University, Pittsburgh, Pennsylvania 15213, USA

${ }^{11}$ Enrico Fermi Institute, University of Chicago, Chicago, Illinois 60637, USA

${ }^{12}$ Comenius University, 84248 Bratislava, Slovakia and Institute of Experimental Physics, 04001 Kosice, Slovakia

${ }^{13}$ Joint Institute for Nuclear Research, RU-141980 Dubna, Russia

${ }^{14}$ Duke University, Durham, North Carolina 27708, USA

${ }^{15}$ Fermi National Accelerator Laboratory, Batavia, Illinois 60510, USA

${ }^{16}$ University of Florida, Gainesville, Florida 32611, USA

${ }^{17}$ Laboratori Nazionali di Frascati, Istituto Nazionale di Fisica Nucleare, I-00044 Frascati, Italy

${ }^{18}$ University of Geneva, CH-1211 Geneva 4, Switzerland

${ }^{19}$ Glasgow University, Glasgow G12 8QQ, United Kingdom

${ }^{20}$ Harvard University, Cambridge, Massachusetts 02138, USA

${ }^{21}$ Division of High Energy Physics, Department of Physics, University of Helsinki, FIN-00014 Helsinki,

Finland and Helsinki Institute of Physics, FIN-00014 Helsinki, Finland

${ }^{22}$ University of Illinois, Urbana, Illinois 61801, USA

${ }^{23}$ The Johns Hopkins University, Baltimore, Maryland 21218, USA

${ }^{24}$ Institut für Experimentelle Kernphysik, Karlsruhe Institute of Technology, D-76131 Karlsruhe, Germany

${ }^{25}$ Center for High Energy Physics: Kyungpook National University, Daegu 702-701, Korea; Seoul

National University, Seoul 151-742, Korea; Sungkyunkwan University, Suwon 440-746, Korea;

Korea Institute of Science and Technology Information, Daejeon 305-806, Korea;

Chonnam National University, Gwangju 500-757, Korea;

Chonbuk National University, Jeonju 561-756, Korea;

and Ewha Womans University, Seoul 120-750, Korea

${ }^{26}$ Ernest Orlando Lawrence Berkeley National Laboratory, Berkeley, California 94720, USA

${ }^{27}$ University of Liverpool, Liverpool L69 7ZE, United Kingdom

${ }^{28}$ University College London, London WC1E 6BT, United Kingdom

${ }^{29}$ Centro de Investigaciones Energeticas Medioambientales y Tecnologicas, E-28040 Madrid, Spain

${ }^{30}$ Massachusetts Institute of Technology, Cambridge, Massachusetts 02139, USA

${ }^{31}$ University of Michigan, Ann Arbor, Michigan 48109, USA

${ }^{32}$ Michigan State University, East Lansing, Michigan 48824, USA

${ }^{33}$ Institution for Theoretical and Experimental Physics, ITEP, Moscow 117259, Russia

${ }^{34}$ University of New Mexico, Albuquerque, New Mexico 87131, USA

${ }^{35}$ The Ohio State University, Columbus, Ohio 43210, USA

${ }^{36}$ Okayama University, Okayama 700-8530, Japan

${ }^{37}$ Osaka City University, Osaka 558-8585, Japan

${ }^{38}$ University of Oxford, Oxford OX1 3RH, United Kingdom

${ }^{39 a}$ Istituto Nazionale di Fisica Nucleare, Sezione di Padova, I-35131 Padova, Italy

${ }^{39 b}$ University of Padova, I-35131 Padova, Italy

${ }^{40}$ University of Pennsylvania, Philadelphia, Pennsylvania 19104, USA

${ }^{41 \mathrm{a}}$ Istituto Nazionale di Fisica Nucleare Pisa, I-56127 Pisa, Italy

${ }^{41 \mathrm{~b}}$ University of Pisa, I-56127 Pisa, Italy

${ }^{41 \mathrm{c}}$ University of Siena, I-53100 Siena, Italy

${ }^{41 \mathrm{~d}}$ Scuola Normale Superiore, I-56127 Pisa, Italy

${ }^{41 \mathrm{e}}$ INFN Pavia, I-27100 Pavia, Italy

${ }^{41 \mathrm{f}}$ University of Pavia, I-27100 Pavia, Italy

${ }^{42}$ University of Pittsburgh, Pittsburgh, Pennsylvania 15260, USA

${ }^{43}$ Purdue University, West Lafayette, Indiana 47907, USA

${ }^{44}$ University of Rochester, Rochester, New York 14627, USA

${ }^{45}$ The Rockefeller University, New York, New York 10065, USA

${ }^{46 \mathrm{a}}$ Istituto Nazionale di Fisica Nucleare, Sezione di Roma 1, I-00185 Roma, Italy

${ }^{46 \mathrm{~b}}$ Sapienza Università di Roma, I-00185 Roma, Italy 
${ }^{47}$ Mitchell Institute for Fundamental Physics and Astronomy, Texas A\&M University, College Station, Texas 77843, USA

${ }^{48 \mathrm{a}}$ Istituto Nazionale di Fisica Nucleare Trieste, I-34012 Trieste, Italy

${ }^{48 \mathrm{~b}}$ Gruppo Collegato di Udine, I-33100 Udine, Italy

${ }^{48 \mathrm{c}}$ University of Udine, I-33100 Udine, Italy

${ }^{48 \mathrm{~d}}$ University of Trieste, I-34127 Trieste, Italy

${ }^{49}$ University of Tsukuba, Tsukuba, Ibaraki 305, Japan

${ }^{50}$ Tufts University, Medford, Massachusetts 02155, USA

${ }^{51}$ University of Virginia, Charlottesville, Virginia 22906, USA

${ }^{52}$ Waseda University, Tokyo 169, Japan

${ }^{53}$ Wayne State University, Detroit, Michigan 48201, USA

${ }^{54}$ University of Wisconsin, Madison, Wisconsin 53706, USA

${ }^{55}$ Yale University, New Haven, Connecticut 06520, USA

(Received 28 April 2015; published 18 August 2015)

We measure the particle-level forward-backward production asymmetry in $b \bar{b}$ pairs with masses $\left(m_{b \bar{b}}\right)$ larger than $150 \mathrm{GeV} / c^{2}$, using events with hadronic jets and employing jet charge to distinguish $b$ from $\bar{b}$. The measurement uses $9.5 \mathrm{fb}^{-1}$ of $p \bar{p}$ collisions at a center-of-mass energy of $1.96 \mathrm{TeV}$ recorded by the CDF II detector. The asymmetry as a function of $m_{b \bar{b}}$ is consistent with zero, as well as with the predictions of the standard model. The measurement disfavors a simple model including an axigluon with a mass of $200 \mathrm{GeV} / c^{2}$, whereas a model containing a heavier $345 \mathrm{GeV} / c^{2}$ axigluon is not excluded.

DOI: 10.1103/PhysRevD.92.032006

PACS numbers: 14.65.Ha

\footnotetext{
Deceased.

${ }^{a}$ Visitor from University of British Columbia, Vancouver, BC V6T 1Z1, Canada.

${ }^{b}$ Visitor from Istituto Nazionale di Fisica Nucleare, Sezione di Cagliari, 09042 Monserrato (Cagliari), Italy.

${ }^{c}$ Visitor from University of California Irvine, Irvine, CA 92697, USA.

${ }^{\mathrm{d}}$ Visitor from Institute of Physics, Academy of Sciences of the Czech Republic, 182 21, Czech Republic.

${ }^{\mathrm{e}}$ Visitor from CERN, CH-1211 Geneva, Switzerland.

${ }^{\mathrm{f}}$ Visitor from Cornell University, Ithaca, NY 14853, USA.

${ }^{\mathrm{g}}$ Visitor from University of Cyprus, Nicosia CY-1678, Cyprus.

${ }^{\mathrm{h}}$ Visitor from Office of Science, U.S. Department of Energy, Washington, DC 20585, USA.

${ }^{\mathrm{i}}$ Visitor from University College Dublin, Dublin 4, Ireland.

${ }^{\mathrm{j}}$ Visitor from ETH, 8092 Zürich, Switzerland.

${ }^{\mathrm{k}}$ Visitor from University of Fukui, Fukui City, Fukui Prefecture, Japan 910-0017.

${ }^{\mathrm{l}}$ Visitor from Universidad Iberoamericana, Lomas de Santa Fe, México, C.P. 01219, Distrito Federal.

${ }^{\mathrm{m}}$ Visitor from University of Iowa, Iowa City, IA 52242, USA.

${ }^{\mathrm{n}}$ Visitor from Kinki University, Higashi-Osaka City, Japan 577-8502.

${ }^{0}$ Visitor from Kansas State University, Manhattan, KS 66506, USA.

${ }^{\mathrm{p}}$ Visitor from Brookhaven National Laboratory, Upton, NY 11973, USA.

${ }^{\mathrm{q}}$ Visitor from Istituto Nazionale di Fisica Nucleare, Sezione di Lecce, Via Arnesano, I-73100 Lecce, Italy.

${ }^{\mathrm{r}}$ Visitor from Queen Mary, University of London, London E1 4NS, United Kingdom.

${ }^{\mathrm{s}}$ Visitor from University of Melbourne, Victoria 3010, Australia.

${ }^{t}$ Visitor from Muons, Inc., Batavia, IL 60510, USA.

${ }^{\mathrm{u}}$ Visitor from Nagasaki Institute of Applied Science, Nagasaki 851-0193, Japan.

${ }^{\mathrm{v}}$ Visitor from National Research Nuclear University, Moscow 115409, Russia.

${ }^{\mathrm{w}}$ Visitor from Northwestern University, Evanston, IL 60208, USA.

${ }^{\mathrm{x}}$ Visitor from University of Notre Dame, Notre Dame, IN 46556, USA.

${ }^{\mathrm{y}}$ Visitor from Universidad de Oviedo, E-33007 Oviedo, Spain.

${ }^{\mathrm{z}}$ Visitor from CNRS-IN2P3, Paris F-75205, France.

${ }^{\text {aa }}$ Visitor from Universidad Tecnica Federico Santa Maria, 110v Valparaiso, Chile.

${ }^{\mathrm{bb}}$ Visitor from Sejong University, Seoul 143-747, Korea.

${ }^{\mathrm{cc}}$ Visitor from The University of Jordan, Amman 11942, Jordan.

${ }^{\mathrm{dd}}$ Visitor from Universite Catholique de Louvain, 1348 Louvain-La-Neuve, Belgium.

${ }^{\text {ee }}$ Visitor from University of Zürich, 8006 Zürich, Switzerland.

${ }^{\mathrm{ff}}$ Visitor from Massachusetts General Hospital, Boston, MA 02114 USA.

${ }^{\mathrm{gg}}$ Visitor from Harvard Medical School, Boston, MA 02114 USA.

${ }^{\text {hh }}$ Visitor from Hampton University, Hampton, VA 23668, USA.

${ }^{\text {ii } V i s i t o r ~ f r o m ~ L o s ~ A l a m o s ~ N a t i o n a l ~ L a b o r a t o r y, ~ L o s ~ A l a m o s, ~ N M ~ 87544, ~ U S A . ~}$

${ }^{\mathrm{jj}}$ Visitor from Università degli Studi di Napoli Federico I, I-80138 Napoli, Italy.
}

Published by the American Physical Society under the terms of the Creative Commons Attribution 3.0 License. Further distribution of this work must maintain attribution to the author(s) and the published article's title, journal citation, and DOI. 


\section{INTRODUCTION}

In recent years, the values of the forward-backward asymmetry $\left(A_{\mathrm{FB}}\right)$ of top-quark-pair production measured at the Tevatron proton-antiproton collider at Fermilab have been consistently larger [1] than those predicted by the standard model (SM) [2-7]. Further study of this phenomenon has led to a number of proposed extensions of the SM [8]. One specific class of such models is the lowmass axigluon [9]. These models include a new axialvector boson (axigluon) with a mass below the $t \bar{t}$ threshold and a natural width broad enough to evade detection in light-quark resonance searches and predict a nonzero forward-backward asymmetry due to the interference between amplitudes mediated by the gluon and the axigluon. In the simplest models, the axigluon has equal couplings to all the quarks. Hence, a stringent test of such models is the measurement of the forward-backward asymmetry of pair production of other quark flavors, such as the bottom.

The forward-backward asymmetry for fermionantifermion production is defined as

$$
A_{\mathrm{FB}}=\frac{n_{F}-n_{B}}{n_{F}+n_{B}}
$$

where $n_{F}$ is the number of events where the fermion is forward of the antifermion in rapidity [10] $\left(\Delta y=y_{b}-\right.$ $y_{\bar{b}}>0$ ) and $n_{B}$ is the number where it is backward $(\Delta y<0)$. This definition is invariant under boosts along the beam axis.

At hadron colliders, $b \bar{b}$ pairs are almost exclusively produced by the strong interaction of quarks and gluons (QCD). The vast majority of $b \bar{b}$ pairs are produced via gluon-gluon fusion, which yields no asymmetry due to the symmetric initial state. In the $q \bar{q} \rightarrow b \bar{b}$ process, a positive $A_{\mathrm{FB}}(b \bar{b})$ arises from higher-order QCD corrections involving either real or virtual gluons. Since the valence-quark parton-density functions dominate over the gluon parton-density functions at large Bjorken $x$, we enhance the contribution of the quark-antiquark initial state over the symmetric gluon-fusion background by requiring large values of $b \bar{b}$ mass, $m_{b \bar{b}}>$ $150 \mathrm{GeV} / c^{2}$.

A number of theoretical predictions of the SM value for $A_{\mathrm{FB}}(b \bar{b})$ at high mass have been reported, using various techniques and kinematic requirements and yielding a range of predictions [4,7,11]. Kühn and Rodrigo [4] computed that the asymmetry for $b \bar{b}$ pairs with $\sqrt{\hat{s}} \geq$ $300 \mathrm{GeV}$ and production angle $\left|\cos \theta^{*}\right|<0.9$ falls in the range $4.3 \%$ to $5.1 \%$. Manohar and Trott [7] computed $A_{\mathrm{FB}}(b \bar{b})$ in several bins of $m_{b \bar{b}}$. They found $A_{\mathrm{FB}}(b \bar{b})=$ $0.4 \%$ inclusively and $A_{\mathrm{FB}}(b \bar{b})=7.8 \%$ to $8.1 \%$ for $350<m_{b \bar{b}}<650 \mathrm{GeV} / c^{2}$, depending on the choice of the factorization and renormalization scales. Grinstein and Murphy [11] computed predictions using a variety of kinematic requirements, including an estimate using our kinematic requirements. The results of this calculation [11] are summarized in Table I.

Models containing a low-mass axigluon that has the same couplings to all quark flavors also predict a forwardbackward asymmetry in bottom-quark-pair production. This asymmetry arises from the interference between the gluon and axigluon in the same fashion as the $Z / \gamma^{*}$ interference of the Drell-Yan process [12]. One key feature of this interference is that the $A_{\mathrm{FB}}$ changes sign at the mass pole of the heavy axial resonance [9]. The observation of such a sign flip would provide a significant indication of the underlying dynamics. The predictions of two different representative axigluon models are given in Table I [9]. A relatively light $200 \mathrm{GeV} / c^{2}$ axigluon has been previously studied in the context of the top-quark asymmetry [13], and a heavier $345 \mathrm{GeV} / c^{2}$ axigluon is of interest because its mass is just below the $t \bar{t}$ threshold. Both of these axigluons are assumed to have a width equal to $25 \%$ of their mass.

The predictions of both the SM and the models of non-SM physics under consideration are estimated at the parton level and do not explicitly include the effects of hadronization.

Although the LHCb experiment has measured a related quantity [14], $A_{\mathrm{FB}}$ can only be measured in protonantiproton collisions [15]. At the Tevatron, the D0 experiment has measured the forward-backward asymmetry of $B^{ \pm}$meson production [16]. The momenta of the $B^{ \pm}$mesons in that measurement constrain the masses of the $b \bar{b}$ pairs to be smaller than the range probed in this measurement.

\section{EXPERIMENTAL APPARATUS AND EVENT SELECTION}

The Fermilab Tevatron is a proton-antiproton collider with a center-of-mass energy $\sqrt{s}=1.96 \mathrm{TeV}$.

TABLE I. Predicted values of $A_{\mathrm{FB}}$ in the standard model [11] and in two models with an axigluon [9]. In the second column, the first contribution to the uncertainty is due to neglected higher-order terms, and the second contribution is due to the combined effect of varying the factorization and renormalization scales. The assumed axigluon mass is listed, and its assumed width is $25 \%$ of the mass in both cases. The selection requirements imposed on all three calculations match the event selection requirements employed in our analysis: pseudorapidity $\left|\eta_{b, \bar{b}}\right|<1.1$.

\begin{tabular}{lccc}
\hline \hline $\begin{array}{l}m_{b \bar{b}} \text { range } \\
\mathrm{GeV} / c^{2}\end{array}$ & $\mathrm{SM}$ & $\begin{array}{c}A_{\mathrm{FB}}(b \bar{b})[\%] \\
200 \mathrm{GeV} / c^{2}\end{array}$ & $345 \mathrm{GeV} / c^{2}$ \\
\hline$[150,225]$ & $2.43 \pm 0.73_{-0.01}^{+0.02}$ & $-2.9_{-0.9}^{+0.4}$ & $-1.9_{-0.9}^{+0.4}$ \\
{$[225,325]$} & $4.61 \pm 1.38_{-0.13}^{+0.15}$ & $20.4_{-1.0}^{+0.7}$ & $-9.9_{-0.6}^{+0.6}$ \\
{$[325,1960]$} & $8.70 \pm 2.61_{-0.51}^{+0.61}$ & $20.2_{-0.6}^{+0.4}$ & $16.4_{-0.9}^{+0.4}$ \\
\hline \hline
\end{tabular}


The CDF II detector [17] is an azimuthally symmetric magnetic spectrometer with a large tracking volume inside a solenoid. Outside the solenoid are sampling calorimeters. The calorimeters are further surrounded by the steel flux return of the solenoid and muon detectors [17].

The data used for this analysis were collected by the CDF II detector using three online event selections (triggers), which require at least one jet with transverse energy $E_{T}>50,70$, or $100 \mathrm{GeV}$, respectively [10]. To control the trigger rate, the two lower-threshold triggers only accept one event out of every 100 or 8 events that satisfy their requirements, respectively, while the highest-threshold trigger accepts all events meeting its requirements. After data-quality requirements and trigger acceptance rates, the integrated luminosities of the samples are $95 \mathrm{pb}^{-1}$, $1.2 \mathrm{fb}^{-1}$, and $9.5 \mathrm{fb}^{-1}$, respectively.

The offline event selection requires at least two jets $[18,19]$ with $E_{T}>20 \mathrm{GeV}$ and pseudorapidity $|\eta|<1.1$. The requirement of $|\eta|<1.1$ restricts the jets to the fiducial region of the silicon tracking and also avoids the transition region between the central and plug calorimeters, so that the trigger is $100 \%$ efficient in the selected region. Of the jets that pass these requirements, exactly two must contain a secondary vertex (separated transversely from the primary $p \bar{p}$ interaction vertex) consistent with the decay of a $b$ quark ( $b$-tagged jets), identified using a secondary-vertex identification algorithm [20]. The observed invariant mass $m_{b \bar{b}}$ of the two $b$-tagged jets is required to be at least $150 \mathrm{GeV} / c^{2}$.

To maximize the statistical significance of the analysis, we separate the data into several subsamples depending on $m_{b \bar{b}}$ (three subsamples, see Sec. III), on the estimated charges of the partons that produced the jets (four subsamples, see Sec. III A), and on the quality of the $b$ tags (three subsamples, see Sec. III C), yielding a total of 36 subsamples.

\section{METHODOLOGY}

We discriminate jets originated from $b$ quarks ( $b$ jets) from jets originated from $\bar{b}$ quarks ( $\bar{b}$ jets) using the momentum-weighted average of the charges of the particles associated with each jet (jet charge),

$$
Q_{\text {jet }}=\frac{\sum_{i} q_{i}\left(\vec{p}_{i} \cdot \vec{p}_{\text {jet }}\right)^{0.5}}{\sum_{i}\left(\vec{p}_{i} \cdot \vec{p}_{\text {jet }}\right)^{0.5}},
$$

where the sum $i$ is over all tracks in the jet, $q_{i}$ is the charge of the corresponding particle, and $\vec{p}_{i}$ and $\vec{p}_{\text {jet }}$ are the momentum vectors of the particle and of the jet, respectively. The exponent 0.5 was chosen to maximize the power of the jet charge in separating $b$ jets from $\bar{b}$ jets [21].

The asymmetry depends on the mass of the $b \bar{b}$ pair, both in the SM and in models with an axigluon. To study the behavior of $A_{\mathrm{FB}}$ as a function of $m_{b \bar{b}}$, we divide the sample into several ranges (bins) of $m_{b \bar{b}}$. The choice of $m_{b \bar{b}}$ bins is motivated by the trigger. Each jet-energy-trigger threshold efficiently selects events only over a limited range of $m_{b \bar{b}}$. Events with $150<m_{b \bar{b}}<225 \mathrm{GeV} / c^{2}$ are selected with a jet transverse-energy threshold of $50 \mathrm{GeV}$, and the thresholds of 70 and $100 \mathrm{GeV}$ are used to select events with $m_{b \bar{b}}$ in the ranges $225<m_{b \bar{b}}<325 \mathrm{GeV} / c^{2}$ and $325 \mathrm{GeV} /$ $c^{2}<m_{b \bar{b}}$, respectively.

The measurement of $A_{\mathrm{FB}}(b \bar{b})$ must account for non- $b \bar{b}$ backgrounds, detector effects, and the dilution of the asymmetry due to misidentification of $b$ jets as $\bar{b}$ jets and vice versa. We therefore define a detector-level $A_{\mathrm{FB}}$,

$$
A_{\mathrm{FB}}^{\mathrm{det}}=\frac{1}{2 P-1} \frac{\left(N_{F}-N_{F}^{\mathrm{bkgd}}\right)-\left(N_{B}-N_{B}^{\mathrm{bkgd}}\right)}{N_{F}-N_{F}^{\mathrm{bkgd}}+N_{B}-N_{B}^{\mathrm{bkgd}}},
$$

where $N_{F}$ and $N_{B}$ are the observed numbers of events, $N_{F}^{\mathrm{bkgd}}$ and $N_{B}^{\mathrm{bkgd}}$ are the estimated numbers of background events, and $P$ is the probability to make the charge assignment correctly. To account for additional effects, such as the finite resolution and acceptance of the CDF II detector, we employ a Bayesian technique to measure the $A_{\mathrm{FB}}$ at the particle level. "Particle level" refers to quantities reconstructed from final-state particles with lifetimes greater than $10 \mathrm{ps}$ [22].

The background levels are determined with a datadriven technique based on the $b$-tagged vertex mass as described in Sec. IIIC. The calibration of the charge misidentification and the calculation of $P$ is presented in Sec. III A, and the background asymmetries are discussed in Sec. III D. Finally, we use a Markov-chain Monte Carlo calculation to derive particle-level results by identifying the maxima of the marginalized posterior probability densities and by constructing associated credible intervals. The correction to the particle level, including the effects of mismeasurement and acceptance, are described in Sec. III G.

\section{A. Identification of the $b$ jet using jet charge}

The forward or backward assignment is performed using the momentum-weighted track charge, or "jet charge" [see Eq. (2)], for each of the two $b$-tagged jets. Distributions of the jet charge $\left(Q_{\text {jet }}\right)$ for $b$ jets and $\bar{b}$ jets are shown in Fig. 1. We use the difference of the two jet charges $\Delta Q=Q_{1}-Q_{2}$ to make the assignment: if $\Delta Q$ is negative, the jet with charge $Q_{1}$ is considered to be the $b$ jet (because the $b$ quark is negatively charged), and if $\Delta Q$ is positive, the jet with charge $Q_{2}$ is considered to be the $b$ jet.

The performance of the jet-charge algorithm is calibrated with data, by dividing the distribution of $Q_{\text {jet }}$ into bins. The bin edges are $-0.25,0$, and 0.25 , for a total of four bins (the lowest- and highest-charge bins are open). We arbitrarily assign $Q_{\text {jet }}$ values of $-0.5,-0.25,0.25$, and 0.5 to the jets 


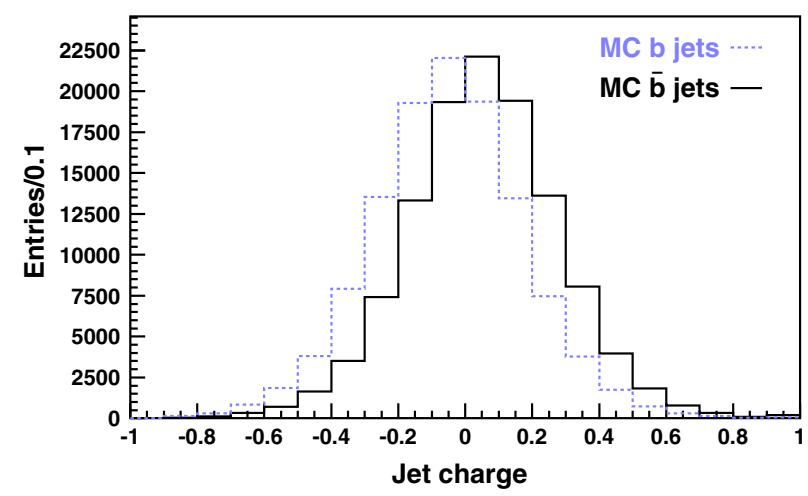

FIG. 1 (color online). Distributions of the charge of $b$ and $\bar{b}$ jets in Monte Carlo simulation of two-jet events.

falling into each respective bin. This translates into five bins of $|\Delta Q|$ with values $0,0.25,0.5,0.75$, and 1.0 . The bin with $|\Delta Q|=0$ is not informative because it gives no indication of how to assign the $b$ jet, so only four bins of $|\Delta Q|$ are used.

\section{B. Charge-identification probability}

The probability $P$ of correctly assigning the $b$ jet and $\bar{b}$ jet, introduced in Eq. (3), is estimated from the data. We evaluate this in each bin of $|\Delta Q|$, so that

$$
\begin{aligned}
P_{0.25} & =\frac{p_{0.5}\left(1-p_{0.25}\right)}{p_{0.5}\left(1-p_{0.25}\right)+\left(1-p_{0.5}\right) p_{0.25}}, \\
P_{0.5} & =\frac{p_{0.25}^{2}}{p_{0.25}^{2}+\left(1-p_{0.25}\right)^{2}}, \\
P_{0.75} & =\frac{p_{0.25} p_{0.5}}{p_{0.25} p_{0.5}+\left(1-p_{0.25}\right)\left(1-p_{0.5}\right)}, \quad \text { and } \\
P_{1.0} & =\frac{p_{0.5}^{2}}{p_{0.5}^{2}+\left(1-p_{0.5}\right)^{2}},
\end{aligned}
$$

where the subscript of $P$ indicates $|\Delta Q|$, and the various $p_{\left|Q_{\text {jet }}\right|}$ are the probabilities that a jet with a binned charge of $\left|Q_{\text {jet }}\right|$ is correctly identified as $b$ or $\bar{b}$ by the sign of $Q_{\text {jet }}$. These expressions are derived by exhaustively considering all the possibilities. For example, $|\Delta Q|=1.0$ implies that one jet has $Q_{\text {jet }}=+0.5$ and the other jet has $Q_{\text {jet }}=-0.5$. If the $b$ jet has charge -0.5 , then the use of $\Delta Q$ allows for a correct assignment of the $b$ and $\bar{b}$ jets. This case occurs with probability $p_{0.5}^{2}$, because both jets (with $\left|Q_{\text {jet }}\right|=0.5$ ) have a jet charge of which the sign matches the sign of the charge of the originating quark. The other possibility is that we misidentify both jets, which occurs with probability $\left(1-p_{0.5}\right)^{2}$. The denominator in Eq. (4) is the sum of these two probabilities, and the numerator only contains the correct case.

To measure $p_{\left|Q_{\mathrm{jet}}\right|}$, we measure the number of oppositecharge events, $N_{\mathrm{OC}}$, and number of same-charge events, $N_{\mathrm{SC}}$, in the data in each $m_{b \bar{b}}$ subsample. These numbers are corrected for the presence of the background using the calibrated $b$-fractions (see Sec. IIIC) and the background model described in Sec. III D. We assume that the remaining sample is composed of $b \bar{b}$ with no contamination from $b b$ or $\bar{b} \bar{b}$ and compute the opposite-charge fraction $F_{\mathrm{OC}}=N_{\mathrm{OC}} /\left(N_{\mathrm{OC}}+N_{\mathrm{SC}}\right)$.

In events in which both jets have $\left|Q_{\text {jet }}\right|=0.25$, the opposite-sign fraction is expressed as

$$
F_{\mathrm{OC}}^{0.25-0.25}=p_{0.25}^{2}+\left(1-p_{0.25}\right)^{2},
$$

where the term $p_{0.25}^{2}$ arises from events in which both $b$ jets have the correct sign, and the term $\left(1-p_{0.25}\right)^{2}$ arises from events in which both $b$ jets have the wrong sign. Similarly, we have

$$
\begin{gathered}
F_{\mathrm{OC}}^{0.25-0.5}=p_{0.25} p_{0.5}+\left(1-p_{0.25}\right)\left(1-p_{0.5}\right), \quad \text { and } \\
F_{\mathrm{OC}}^{0.5-0.5}=p_{0.5}^{2}+\left(1-p_{0.5}\right)^{2},
\end{gathered}
$$

for events containing one jet with $\left|Q_{\text {jet }}\right|=0.25$ and one jet with $\left|Q_{\text {jet }}\right|=0.5$ and for events in which both jets have $\left|Q_{\text {jet }}\right|=0.5$, respectively. We measure each $F_{\mathrm{OC}}$ value in the data and solve for each $p_{\left|Q_{\text {jet }}\right|}$ and then for each of the four $P_{|\Delta Q|}$.

\section{Sample purity}

Eq. (3) also requires knowledge of the rate of background events, $N_{F}^{\mathrm{bkgd}}$ and $N_{B}^{\mathrm{bkgd}}$. We obtain the background yields by estimating the number of $b \bar{b}$ events $N_{b \bar{b}}$ and subtracting it from the number of events observed.

We divide the data into subsamples with varying $b$-tag quality, which in turn provides subsamples of varying $b \bar{b}$ purity and improves the statistical power of the measurement. Since we apply $b$-tags to jets based on the presence of a secondary vertex, the quality of the $b$-tag is based on the confidence level of the identification of the secondary vertex. Specifically, the significance is based on the distance $L_{2 \mathrm{D}}$, in the plane perpendicular to the beam, between the primary and secondary vertices, projected onto the jet momentum [20]. Jets with a significance $\left[\left|L_{2 \mathrm{D}}\right| / \sigma\left(L_{2 \mathrm{D}}\right)\right]$ greater than 20 are high significance or " $\mathrm{H}$ " tags, and jets with a lower significance are "L" tags. This results in the following independent subsamples ranked in order of increasing $b \bar{b}$ purity: LL (both jets are L tagged), LH (one $\mathrm{L}$ and one $\mathrm{H}$ tag), and $\mathrm{HH}$ (both $\mathrm{H}$ tags).

We estimate the number of events in which both $b$-tagged jets are genuine $b$ jets by counting events in which one or both of the $b$-tagged jets have a negative $L_{2 \mathrm{D}}$. These "negative" tags are predominantly false tags from light-flavor jets and are a consequence of the finite position resolution of the tracking system. We expect the rate of false tags from this source to be equal for positive and negative tags. There are additional false positive tag contributions from hyperon decays and from interactions 
between high-momentum particles and the detector material. This results in an excess of positive over negative false tags. We exploit this relationship between the number of positively and negatively tagged light-flavor jets and compute the number of true $b \bar{b}$ events using

$$
N_{b \bar{b}}=\frac{1}{\xi}\left(N_{++}-\lambda N_{+-}+\lambda^{2} N_{--}\right),
$$

where $N_{++}$is the number of observed positive double-tag events, $N_{+-}$is the number of events with one of the tags negative, $N_{--}$is the number with both tags negative, $\lambda$ is the ratio of positive to negative false tags, and $\xi=$ $1-2 \lambda r+\lambda^{2} r^{2}$ is a factor that accounts for the presence of negatively tagged $b$ jets. Here, $r=\epsilon_{b}^{-} / \epsilon_{b}^{+}$is the ratio of the negative to positive tag efficiencies for $b$ jets, estimated using Monte Carlo (MC) simulation and data. Finally, $N_{b \bar{b}}$ is corrected by a scale factor derived from $\mathrm{MC}$ to account for a bias resulting from the presence of charm jets, which is expected to be less than $10 \%$ of the $b \bar{b}$ component [23].

We measure the ratio of positive to negative light-flavor tag rates $\lambda$ following Ref. [23]. Since the MC indicates no strong dependence of $\lambda$ on jet $E_{T}$, we use the same value for all three dijet mass bins. We study the distribution of the invariant mass of all charged particles associated with the secondary vertex, $m_{\mathrm{VTX}}$ [20], for both negative and positive tags. We subtract the negative tag distribution from the positive tag distribution, yielding the "net" distribution. The relative rates of MC-derived templates for $b, c$ and light-flavor jets, left as free parameters, are fit in the net $m_{\mathrm{VTX}}$ distribution observed in the data (see Fig. 2).

These net flavor fractions allow for the normalization of the complete positive- and negative-tagged distributions of $m_{\mathrm{VTX}}$ for all jet flavors. We find that the negativetagged component of the MC templates must be scaled by 1.5 (1.3) to match the total number of low (high) significance negative tags observed in the data. This scaling is propagated into the positive-tagged region of the templates as this population of "fake" tags is considered to be positive-negative symmetric, leaving the original net distributions unchanged [20]. From these corrected templates, we then obtain $\lambda$ for low- and highsignificance $b$-tagged jets. We also correct the values of $r$ used in the three dijet mass bins for the higher negative tag rates observed in the data.

\section{Background model}

The non- $b \bar{b}$ background includes a wide variety of physics processes, such as $b+\operatorname{mistag}, u \bar{u}, d \bar{d}$, gluon jets, etc. Of primary concern is the scattering of the valence quarks, which proceeds through the $t$-channel exchange of a gluon and exhibits a forward-backward asymmetry due to the forward Rutherford peak. Rather than attempting to
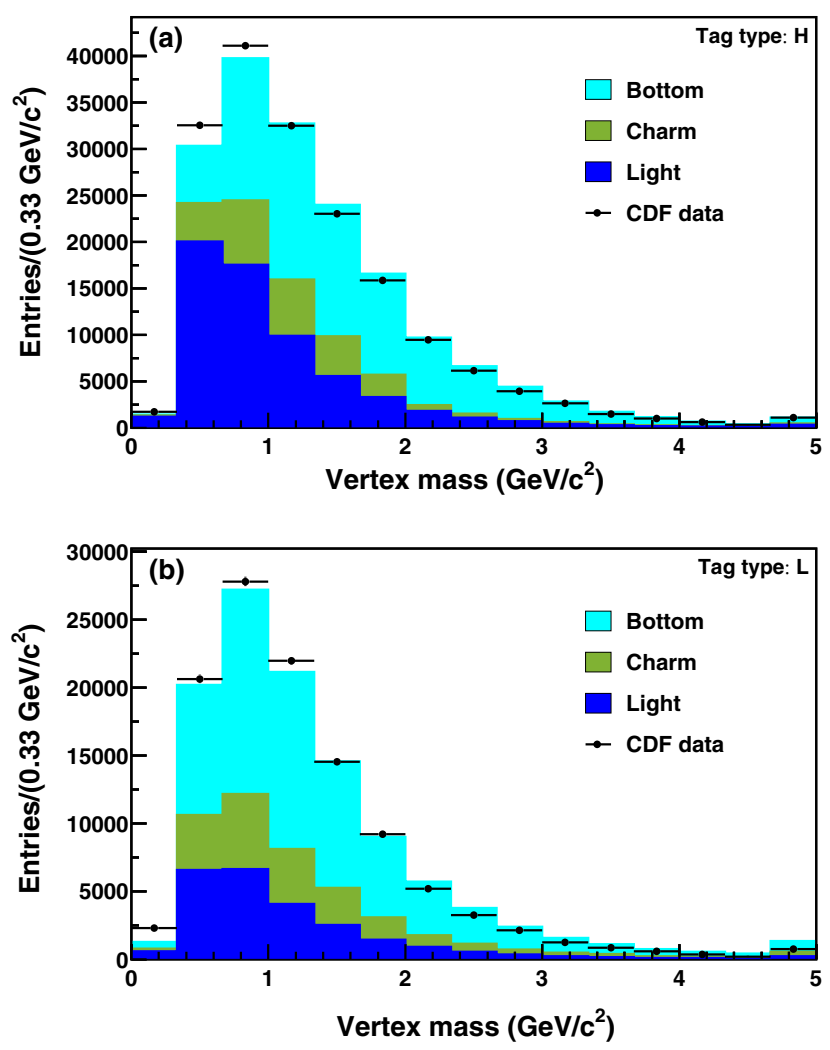

FIG. 2 (color online). Distribution of net $m_{\mathrm{VTX}}$, for $\mathrm{H}$ (a) and $\mathrm{L}$ (b) tagged data, with the fit overlaid. The negative tag rates are subtracted from the positive tag rates. The histograms are stacked on top of one another.

tune the simulation to reproduce all of these processes in the proper amounts, we use data in a control region that is expected to be enhanced in the background and depleted of the $b \bar{b}$ signal. The control region is defined by loosening the requirements of the $b$-tagging algorithm (looser than the low-significance " $\mathrm{L}$ " tag) and requiring that at least one of the tagged jets is negatively tagged. The sample of events satisfying these criteria is nonoverlapping with the signal sample and is composed almost entirely of the background but is kinematically very similar to the signal sample. The forward-backward asymmetry of this sample is assumed to approximate the forward-backward asymmetry of the background in the signal sample (see Fig. 3).

The asymmetry of the background is negative, consistent with $u-\bar{u}$ scattering as the most important component. The $u$ quark tends to follow the incoming proton direction in $t$-channel scattering which would seem to yield a positive $A_{\mathrm{FB}}$, but the opposite charge of the $u$ and $b$ quarks, combined with the definition of the asymmetry in terms of the jet charge, reverses the sign of the asymmetry.

Together with the background yields, the estimated background asymmetries are used to estimate the number of forward and backward background events, $N_{F}^{\mathrm{bkgd}}$ and $N_{B}^{\mathrm{bkgd}}$, in Eq. (3). 


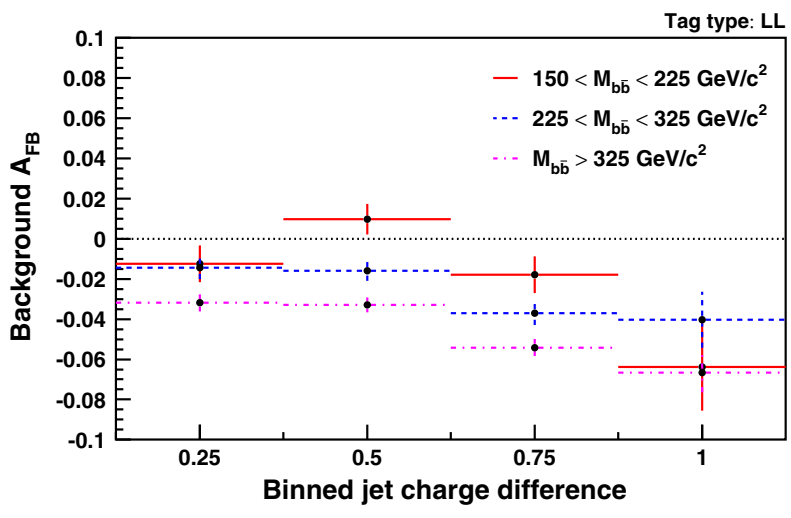

FIG. 3 (color online). Estimated asymmetry of the backgrounds in each $m_{b \bar{b}}$ bin with tag type LL, as a function of $|\Delta Q|$.

\section{E. Jet energy mismeasurement}

Although we correct the energy of each jet for known effects [19], the energy of a measured jet does not exactly match the energy of the corresponding particle-level jet [22]. Mismeasurement of jet energies principally affects the measurement of the dijet mass. Since we use wide mass bins, this effect is small. We estimate the effect using MC samples of dijet events produced with the PYTHIA event generator at leading order in the strong coupling constant, using the CTEQ5L parton distribution functions. We select events that have a pair of bottom quarks, whether produced directly or in the parton shower, to produce the signal model.

This signal model allows the determination of a matrix relating the measured dijet mass to the particle-level dijet mass, as a function of $|\Delta Q|$. The matrix, summed over $|\Delta Q|$, is shown in Fig. 4. In $80 \%$ to $95 \%$ of events, the dijet mass is reconstructed in the same bin. The relative uncertainty on each element of the matrix ranges from a few percent on the diagonal up to very large values far off the diagonal. The

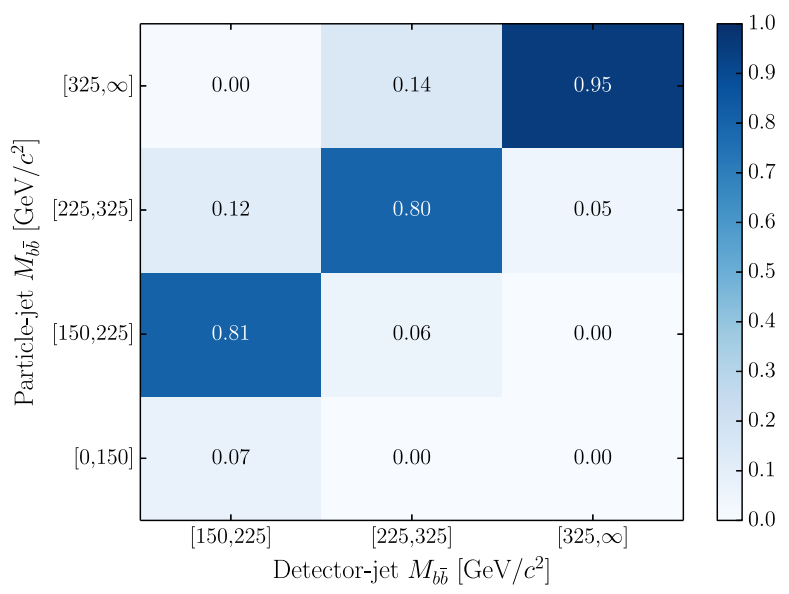

FIG. 4 (color online). Smearing matrix relating measured dijet mass to particle-level dijet mass. The matrix is evaluated from $\mathrm{MC}$, and depends on $|\Delta Q|$, but it is shown summed over bins of $|\Delta Q|$. fraction of events in which the detector-level dijet mass migrates to a different bin than that of the particle-level dijet mass necessitates a correction and increases the uncertainty in the measurement of the particle-level $A_{\mathrm{FB}}$ as a function of dijet mass (see Sec. III G). The correction relies on the MC description of the particle-level dijet mass to be approximately correct. Figure 5 shows that the detector-level dijet mass is correctly described by the $\mathrm{MC}$, which supports the reliability of the smearing matrix.
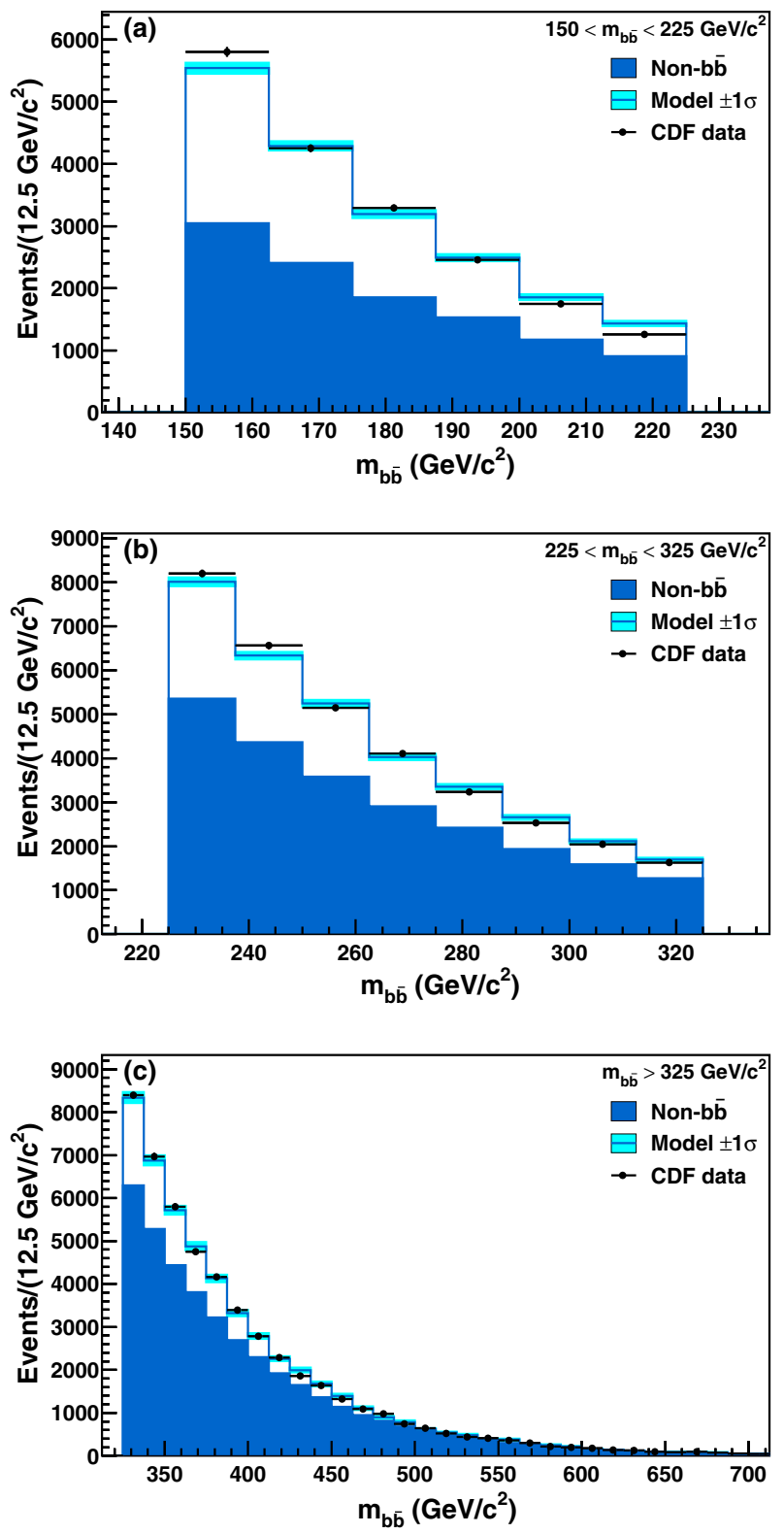

FIG. 5 (color online). Detector-level dijet mass spectra in each of the three $m_{\underline{b} \bar{b}}$ subsamples, showing the spectrum resulting from the non- $b \bar{b}$ component only as well as from the sum of the $b \bar{b}$ and non $-b \bar{b}$ components ("Model $\pm 1 \sigma$ "). The agreement between data and simulation is good, suggesting that the particlelevel dijet mass in $\mathrm{MC}$ simulation and the smearing matrix are also correct within the uncertainties. The non $-b \bar{b}$ component is not included in the smearing matrix. 


\section{F. Selection effects}

We also estimate the effect of the potentially asymmetric acceptance of the detector and analysis selection. We apply the analysis event selection to the simulated events to estimate the fractions of forward $\left(\epsilon_{F}\right)$ and backward $\left(\epsilon_{B}\right)$ events that pass the analysis selection. We perform this estimation in each bin of particle-level dijet mass. Only the ratio of the forward to the backward acceptance affects the analysis, so we compute the ratio $R=\epsilon_{F} / \epsilon_{B}$. We also estimate the uncertainty on this ratio by varying the renormalization and factorization scales used in the signal model by a factor of 2 and the jet-energy scale within its uncertainty.

\section{G. Extraction of the particle-level asymmetry}

To infer the asymmetry at the particle level, we construct a Bayesian model to describe the data. This model combines all effects discussed in the preceding sections, propagates the uncertainties, and allows the data to constrain the uncertainties when possible. The parameters of the model and their assumed prior probability distributions are as follows:

(1) $f_{M Q T}$ is the $b \bar{b}$ fraction in each bin of detector-level dijet mass $M$, charge difference $Q$, and tag quality $T$. The prior probability distribution for $f$ is a normal distribution centered at the calibrated value, with a width equal to the residual uncertainty from the calibration.

(2) $P_{M Q}$ is the correct-charge probability described in Eq. (4). The priors for $p_{0.5}$ and $p_{0.25}$ are normal distributions with the mean and uncertainty taken from the calibration.

(3) $F_{M O T}^{\mathrm{bkgd}}$ and $B_{M O T}^{\mathrm{bkgd}}$ are the rates of forward and backward events in the background-dominated sideband. From these, we calculate the background asymmetry, which is assumed to be consistent with the asymmetry of the background in the signal region. The prior for each of these is the gamma distribution.

(4) $J$ is the shift in the jet energy scale. We coherently shift all of the jet energies in every MC event by $J$ times the jet-energy uncertainty [19]. The prior is a normal distribution with a mean of zero and standard deviation of 1.

(5) $S_{M^{\prime} M Q}(J)$ is a matrix describing the contribution of events with particle-level dijet mass $M^{\prime}$ to the various bins of measured dijet mass and charge difference. The matrix is a function of $J$. The prior is taken from the rate and uncertainty in simulation, and the matrix is normalized so that $\sum_{M^{\prime}} S_{M^{\prime} M Q}(J)=1$.

(6) $\sigma_{M Q T}$ is the rate of events in each bin of detectorlevel $b \bar{b}$ mass, charge difference, and tag quality. This parameter has a uniform prior over the nonnegative range. This parameter is necessary because the simulation does not accurately predict the overall event rate as a function of mass, charge difference, and tag quality.
(7) $R_{M^{\prime}}$ is the ratio of the forward to the backward acceptance. The prior is a normal distribution with mean and width taken from the calibration described in Sec. III F.

(8) $A_{M^{\prime}}$ is the $b \bar{b}$ asymmetry in bins of particle-level mass. This is the parameter we wish to measure. We use a uniform prior from $[-1,1]$.

(9) $A_{M^{\prime}}^{\text {acc }}$ is the $b \bar{b}$ asymmetry after acceptance and selection effects. It is a function of $A_{M^{\prime}}$ and $R_{M^{\prime}}$,

$$
A_{M^{\prime}}^{\mathrm{acc}}=\frac{R_{M^{\prime}}\left(1+A_{M^{\prime}}\right)-\left(1-A_{M^{\prime}}\right)}{R_{M^{\prime}}\left(1+A_{M^{\prime}}\right)+\left(1-A_{M^{\prime}}\right)} .
$$

We compute the rate $\theta$ of forward and backward events expected in the data and compare this rate to that observed in the data (see Table II) via a Poisson likelihood, given an observation of $k$ events, $\left[L(\theta \mid k)=\theta^{k} e^{-\theta} / k !\right]$. The rates are

$$
\begin{aligned}
\theta_{M Q T}^{\text {Forward }}= & {\left[f_{M Q T} \sum_{M^{\prime}} \frac{1+A_{M^{\prime}}^{\mathrm{acc}}\left(2 P_{M Q}-1\right)}{2} S_{M^{\prime} M Q}(J)\right.} \\
& \left.+\left(1-f_{M Q T}\right) \frac{F_{M Q T}^{\mathrm{bkgd}}}{F_{M Q T}^{\mathrm{bkgd}}+B_{M Q T}^{\mathrm{bkgd}}}\right] \times \sigma_{M Q T},
\end{aligned}
$$

and

$$
\begin{aligned}
\theta_{M Q T}^{\text {Backward }}= & {\left[f_{M Q T} \sum_{M^{\prime}} \frac{1-A_{M^{\prime}}^{\mathrm{acc}}\left(2 P_{M Q}-1\right)}{2} S_{M^{\prime} M Q}(J)\right.} \\
& \left.+\left(1-f_{M Q T}\right) \frac{B_{M Q T}^{\mathrm{bkgd}}}{F_{M Q T}^{\mathrm{bkgd}}+B_{M Q T}^{\mathrm{bkgd}}}\right] \times \sigma_{M Q T} .
\end{aligned}
$$

The prior probability densities described above, together with this likelihood, fully specify the posterior probability density for the parameters. To estimate this posterior

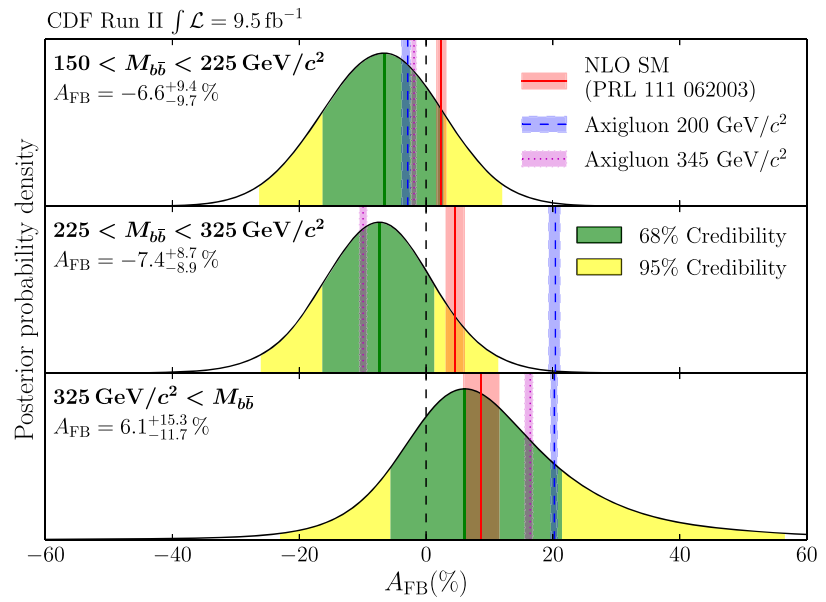

FIG. 6 (color online). Marginal posterior probability distribution of asymmetry in each bin of particle-level $b \bar{b}$ mass. The inner and outer bands represent the $68 \%$ and $95 \%$ credible intervals, respectively. 
density, we employ Markov-chain Monte Carlo sampling [24]. This technique provides us with samples from the posterior probability distribution over the parameter space. The prior probability distributions for the nuisance parameters are quite similar in all cases to the corresponding posterior. We marginalize the nuisance parameters and obtain the posterior density for $A_{M^{\prime}}$, the asymmetry in each bin of particle-level $b \bar{b}$ mass. The marginal distributions are shown in Fig. 6, and the marginalized samples are provided in the Supplemental Material [25].

\section{RESULTS AND CONCLUSIONS}

To characterize the posterior and describe the measurement, we find the highest probability-density credible intervals at $68 \%$ and $95 \%$ credibility for $A_{M^{\prime}}$ in each particle-level mass bin. The posterior densities, along with

TABLE II. Numbers of events observed in the various subsamples.

\begin{tabular}{|c|c|c|c|c|}
\hline$m_{b \bar{b}}$ range & $|\Delta Q|$ & Tag type & $N(\Delta y>0)$ & $N(\Delta y<0)$ \\
\hline \multirow[t]{12}{*}[150,225]{} & \multirow[t]{3}{*}{0.25} & LL & 483 & 472 \\
\hline & & LH & 774 & 841 \\
\hline & & $\mathrm{HH}$ & 465 & 469 \\
\hline & \multirow[t]{3}{*}{0.5} & LL & 734 & 699 \\
\hline & & $\mathrm{LH}$ & 1223 & 1240 \\
\hline & & $\mathrm{HH}$ & 682 & 691 \\
\hline & \multirow[t]{3}{*}{0.75} & LL & 460 & 474 \\
\hline & & LH & 854 & 882 \\
\hline & & $\mathrm{HH}$ & 483 & 519 \\
\hline & \multirow[t]{3}{*}{1.0} & LL & 97 & 81 \\
\hline & & LH & 130 & 141 \\
\hline & & $\mathrm{HH}$ & 101 & 106 \\
\hline \multirow[t]{12}{*}[225,325]{} & \multirow[t]{3}{*}{0.25} & LL & 1014 & 984 \\
\hline & & LH & 1419 & 1520 \\
\hline & & $\mathrm{HH}$ & 686 & 735 \\
\hline & \multirow[t]{3}{*}{0.5} & LL & 1465 & 1499 \\
\hline & & LH & 2197 & 2230 \\
\hline & & $\mathrm{HH}$ & 951 & 992 \\
\hline & \multirow[t]{3}{*}{0.75} & LL & 915 & 979 \\
\hline & & LH & 1455 & 1558 \\
\hline & & $\mathrm{HH}$ & 712 & 735 \\
\hline & \multirow[t]{3}{*}{1.0} & LL & 140 & 160 \\
\hline & & LH & 293 & 256 \\
\hline & & $\mathrm{HH}$ & 149 & 140 \\
\hline \multirow[t]{12}{*}[325,1960]{} & \multirow[t]{3}{*}{0.25} & LL & 1565 & 1636 \\
\hline & & $\mathrm{LH}$ & 2214 & 2401 \\
\hline & & $\mathrm{HH}$ & 965 & 937 \\
\hline & \multirow[t]{3}{*}{0.5} & LL & 2228 & 2379 \\
\hline & & LH & 3196 & 3254 \\
\hline & & $\mathrm{HH}$ & 1298 & 1318 \\
\hline & \multirow[t]{3}{*}{0.75} & LL & 1585 & 1680 \\
\hline & & LH & 2286 & 2512 \\
\hline & & $\mathrm{HH}$ & 958 & 963 \\
\hline & \multirow[t]{3}{*}{1.0} & LL & 253 & 281 \\
\hline & & LH & 396 & 439 \\
\hline & & $\mathrm{HH}$ & 189 & 191 \\
\hline
\end{tabular}

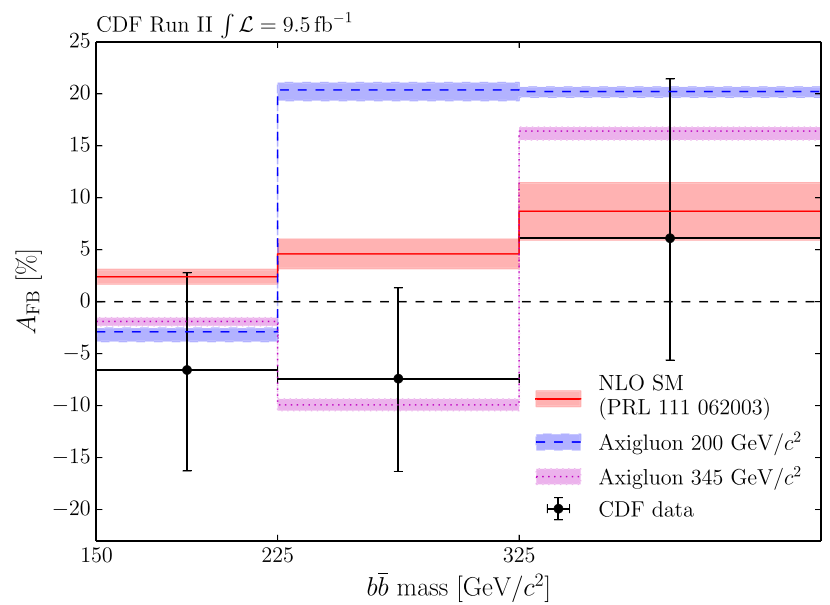

FIG. 7 (color online). Values of maximum a posteriori signal asymmetry as a function of $b \bar{b}$ mass. The error bars represent the $68 \%$ credible intervals.

the intervals describing them, are shown in Fig. 6. The red vertical bands (with solid lines) represent the theoretical predictions from the SM [11], while the blue and magenta bands (with dashed and dotted lines, respectively) represent the predictions from two axigluon models (see Table I). Although the measurement is performed at the particle level, the predictions are at the parton level and do not include the effects of hadronization. Because hadronization effects are not expected to be large, we do not hesitate to interpret the results.

The measured asymmetries, summarized in Fig. 7, are

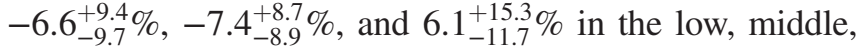
and high $m_{b \bar{b}}$ bins, respectively. These results, which account for the effects of backgrounds, charge misidentification, detector resolution, and nonuniform detector acceptance, are consistent with zero and with the standard model prediction [11] in each bin. Only $0.24 \%$ of the posterior probability density in the middle mass bin has an $A_{\mathrm{FB}}$ larger than predicted by the lighter axigluon model [9] with a mass of $200 \mathrm{GeV} / c^{2}$. Accounting for the lookelsewhere effect following Ref. [26], this is sufficient to exclude the lighter axigluon at more than 95\%. The measurement is unable to exclude the heavier axigluon with a mass of $345 \mathrm{GeV} / c^{2}$. This measurement reduces the allowed parameter space for light axigluon models used to explain the top-quark forward-backward asymmetry.

\section{ACKNOWLEDGMENTS}

We thank the Fermilab staff and the technical staffs of the participating institutions for their vital contributions. This work was supported by the U.S. Department of Energy and National Science Foundation; the Italian Istituto Nazionale di Fisica Nucleare; the Ministry of Education, Culture, Sports, Science and Technology of Japan; the Natural Sciences and Engineering Research Council of Canada; 
the National Science Council of the Republic of China; the Swiss National Science Foundation; the A. P. Sloan Foundation; the Bundesministerium für Bildung und Forschung, Germany; the Korean World Class University Program, the National Research Foundation of Korea; the Science and Technology Facilities Council and the Royal
Society, United Kingdom; the Russian Foundation for Basic Research; the Ministerio de Ciencia e Innovación, and Programa Consolider-Ingenio 2010, Spain; the Slovak R\&D Agency; the Academy of Finland; the Australian Research Council; and the EU community Marie Curie Fellowship Contract No. 302103.
[1] T. Aaltonen et al. (CDF Collaboration), Phys. Rev. D 87, 092002 (2013); V. M. Abazov et al. (D0 Collaboration), Phys. Rev. D 90, 072011 (2014).

[2] M. Czakon, P. Fiedler, and A. Mitov, Phys. Rev. Lett. 115, 052001 (2015).

[3] W. Bernreuther and Z.-G. Si, Phys. Rev. D 86, 034026 (2012).

[4] J. H. Kühn and G. Rodrigo, Phys. Rev. D 59, 054017 (1999).

[5] W. Hollik and D. Pagani, Phys. Rev. D 84, 093003 (2011).

[6] J. H. Kühn and G. Rodrigo, J. High Energy Phys. 01 (2012) 063.

[7] A. V. Manohar and M. Trott, Phys. Lett. B 711, 313 (2012).

[8] Q.-H. Cao, D. McKeen, J. L. Rosner, G. Shaughnessy, and C. E. M. Wagner, Phys. Rev. D 81, 114004 (2010); M. I. Gresham, I.-W. Kim, and K. M. Zurek, Phys. Rev. D 83, 114027 (2011); S. Jung, A. Pierce, and J. D. Wells, Phys. Rev. D 83, 114039 (2011); J. F. Kamenik, J. Shu, and J. Zupan, Eur. Phys. J. C 72, 2102 (2012).

[9] J. Aguilar-Saavedra and M. Perez-Victoria, Phys. Lett. B 705, 228 (2011); C. Gross, G. Marques Tavares, M. Schmaltz, and C. Spethmann, Phys. Rev. D 87, 014004 (2013); A. Carmona, M. Chala, A. Falkowski, S. Khatibi, M. M. Najafabadi, G. Perez, and J. Santiago, J. High Energy Phys. 07 (2014) 005; S. Ipek, Phys. Rev. D 87, 116010 (2013).

[10] We use a cylindrical coordinate system with the origin at the center of the CDF II detector; $z$ pointing in the direction of the proton beam; $\theta$ and $\phi$ representing the polar and azimuthal angles, respectively; and pseudorapidity and rapidity defined by $\eta=-\ln \tan (\theta / 2)$ and $y=$ $(1 / 2) \ln \left(E+p_{z} / E-p_{z}\right)$, respectively. The transverse momentum $p_{T}$ (transverse energy $E_{T}$ ) is defined to be $p \sin \theta$ $(E \sin \theta)$.
[11] B. Grinstein and C. W. Murphy, Phys. Rev. Lett. 111, 062003 (2013); C. W. Murphy, arXiv:1504.02493.

[12] T. A. Aaltonen et al. (CDF Collaboration), Phys. Rev. D 89, 072005 (2014); S. Drell and T.-M. Yan, Phys. Rev. Lett. 25, 316 (1970).

[13] T. Aaltonen et al. (CDF Collaboration), Phys. Rev. D 88, 072003 (2013).

[14] R. Aaij et al. (LHCb Collaboration), Phys. Rev. Lett. 113, 082003 (2014).

[15] The forward-backward asymmetry must vanish in a symmetric proton-proton initial state.

[16] V. M. Abazov et al. (D0 Collaboration), Phys. Rev. Lett. 114, 051803 (2015).

[17] D. E. Acosta et al. (CDF Collaboration), Phys. Rev. D 71, 032001 (2005).

[18] We use a fixed-size cone algorithm to identify jets with a cone radius $\Delta R=\sqrt{(\Delta \eta)^{2}+(\Delta \phi)^{2}}=0.7$.

[19] A. Bhatti et al., Nucl. Instrum. Methods Phys. Res., Sect. A 566, 375 (2006).

[20] D. Acosta et al. (CDF Collaboration), Phys. Rev. D 71, 052003 (2005).

[21] T. Aaltonen et al., Phys. Rev. D 88, 032003 (2013).

[22] T. Aaltonen et al. (CDF Collaboration), Phys. Rev. D 78, 052006 (2008).

[23] T. Aaltonen et al. (CDF Collaboration), Phys. Rev. D 85, 032005 (2012).

[24] A. Patil, D. Huard, and C. J. Fonnesbeck, J. Stat. Software 35, 1 (2010).

[25] See Supplemental Material at http://link.aps.org/ supplemental/10.1103/PhysRevD.92.032006, for marginalized samples from the posterior probability distribution.

[26] Z. Šidák, J. Am. Stat. Assoc. 62, 626 (1967). 